\title{
Correction to: Assessment of the efficacy of teriparatide treatment for osteoporosis on lumbar fusion surgery outcomes: a systematic review and meta-analysis
}

\author{
Nida Fatima ${ }^{1}$ D $\cdot$ Elie Massaad $^{1} \cdot$ Muhamed Hadzipasic $^{1} \cdot$ Ganesh M. Shankar $^{1} \cdot$ John H. Shin $^{1}$ \\ Published online: 13 November 2021 \\ ๑) Springer-Verlag GmbH Germany, part of Springer Nature 2021
}

Correction to: Neurosurg Rev (2021) 44:1357-1370

https://doi.org/10.1007/s10143-020-01359-3

The authors regret that the version of Figure 2 that appears in the original article is incorrect.

The original article can be found online at https://doi.org/10.1007/ s10143-020-01359-3.

Nida Fatima

Fnida99@yahoo.com

1 Department of Neurosurgery, Massachusetts General

Hospital, Harvard Medical School, Boston, MA, USA 
The correct Figure 2 appears below.

a

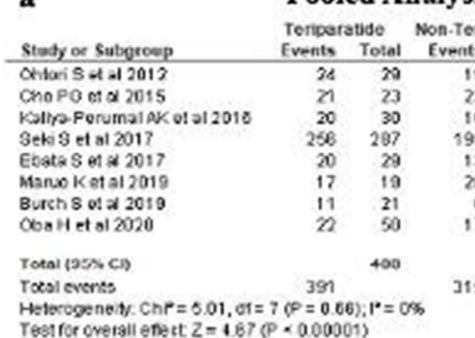

Pooled Analysis of Fusion Rate

$$
\text { Odds Rato }
$$

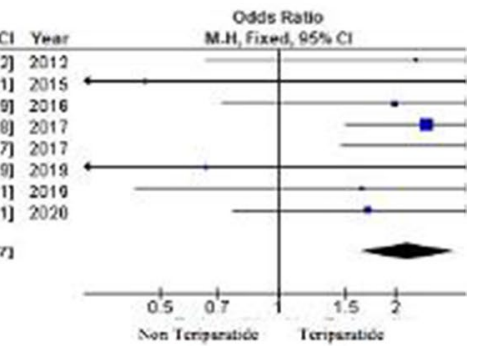

Non Teripuratide Teripantide

\section{b}

\section{Pooled Analysis of Fusion Rate}

\begin{tabular}{|c|c|c|c|c|c|c|c|c|c|c|}
\hline \multirow[b]{2}{*}{ Study or Subgroup } & \multicolumn{2}{|c|}{ Teriparatide } & \multicolumn{2}{|c|}{ Bisphosphonate } & \multicolumn{3}{|c|}{ Odas Ratio } & \multirow{2}{*}{\multicolumn{3}{|c|}{$\begin{array}{l}\text { Odds Ratio } \\
\text { M-H. Fixed, } 95 \mathrm{Cl}\end{array}$}} \\
\hline & Events & Total & Events & Total & Weight & M-H. Fixed, 95N Ca & Year & & & \\
\hline Ohtoti $S$ ot al 2012 & 24 & 28 & 18 & 28 & $80 \%$ & $227[0.85,7.97]$ & 2012 & & & \\
\hline Cho PO et al 2015 & 21 & 23 & 23 & 24 & $5.3 \%$ & $0.86[0.04,5.41]$ & 2015 & 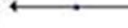 & & \\
\hline Saks S e12a12017 & 256 & 287 & 195 & 252 & $608 \%$ & $2.41[1.50,3.88]$ & 2017 & & & \\
\hline Oba Hetal 2020 & 22 & 60 & 17 & 54 & 24.86 & $171[0.77,3.81]$ & 2020 & & & \\
\hline Total (25^ C) & & 389 & & 358 & $100.0 \%$ & $2.12[1.45,3.11]$ & & & & \\
\hline Tctal everts & 323 & & 254 & & & & & & & \\
\hline $\begin{array}{l}\text { Heterogen eily. Chi" } \\
\text { Test for overal enfext }\end{array}$ & $\begin{array}{l}2.06, \mathrm{df}= \\
z=3.87\end{array}$ & $\begin{array}{l}3 P=0 \\
=0.00\end{array}$ & $\begin{array}{l}56) ; 1^{2}=08 \\
01)\end{array}$ & & & & & 0.5 & 0.7 & 1.5 \\
\hline
\end{tabular}

c

\section{Pooled Analysis of Fusion Rate}

\begin{tabular}{|c|c|c|c|c|c|c|c|c|c|c|}
\hline Study of Suboroup & \multicolumn{2}{|c|}{ Teriparasde } & \multicolumn{2}{|c|}{ Placebo } & \multicolumn{3}{|c|}{ OSAs Rotio } & \multicolumn{3}{|c|}{$\begin{array}{l}\text { Odds Ratio } \\
\text { M.H. Fixed, } 95 \% \mathrm{Cl}\end{array}$} \\
\hline Maruo K et al 2018 & 17 & 18 & 26 & 28 & $15.5 \%$ & $0.65\{0.08,5.02\}$ & 2018 & & & \\
\hline Eurchs ot al 2019 & 11 & 21 & 6 & 15 & 23.48 & $1.65[0.43,6.31]$ & 2019 & & & 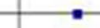 \\
\hline Ebses 5 et al 2013 & 20 & 29 & 13 & 37 & $24.9 \%$ & $8.10[1.46,11.57]$ & 2017 & & & \\
\hline Kolicy.Porumal AK ot al 2016 & 20 & 30 & 16 & 32 & 36.28 & $2.00|0.72,5.52|$ & 2016 & & & \\
\hline Total $(95 \%, C)$ & & 99 & & 112 & $100,0 \%$ & $2.23[1.22,4.08]$ & & & & \\
\hline Total ewonts & 68 & & 81 & & & & & & & \\
\hline $\begin{array}{l}\text { Heterogeneity ChP = } 2.34 \text {, dr } \\
\text { Test por overal efect } Z=2.61\end{array}$ & $\begin{array}{l}3(P=0.4 \\
=0009)\end{array}$ & $0, p=0$ & & & & & & 0.2 & os & 1 \\
\hline
\end{tabular}

Publisher's note Springer Nature remains neutral with regard to jurisdictional claims in published maps and institutional affiliations. 